reveals chalky white pallor of the papilla, without the slightest appearance of shrinkage. I have seen several cases of this kind and they are equally typical.

(To be continued).

\title{
ANNOTATIONS
}

\section{- Mansion House Meeting on Hospital Finance}

Under the auspices of King Edward's Hospital Fund for London, a meeting of representatives of all the London Hospitals took place at the Mansion House on Wednesday, July 6, 1921, Lord Stuart of Wortley in the chair.

The object of the conference was to discuss the three schemes of "mass contributions" referred to by Lord Cave's Committee as likely to provide the key to the problem of hospital finance, namely : (A) Organized weekly contributions of wage-earners on a large scale, but without any definite insurance system; (B) the Oxford scheme, under which the contributors pay $2 \mathrm{~d}$. per week, and are entitled to free treatment; whereas non-contributors are expected to pay; (C) the Sussex scheme, under which, in the modified form proposed for London, the contributor pays $5 \mathrm{~d}$. per week and the hospital gets about as much as under the "Oxford" scheme, the payment also providing the members with other facilities, such as private consultations, dental treatment, etc.

Lord Cave's Committee gave two years for the re-establishment of the finances of the hospitals, but that pre-supposed the acceptance of their recommendation of a Government emergency grant of $£ 1,000,000$ for the first year, and possibly less in the second, whereas the Government had only suggested $£ 500,000$ for the first year conditional on the hospitals taking for themselves all possible steps to get over their financial difficulty. In other words, adopting one of the above schemes in some form or other.

Most of the representatives of the hospitals present were strongly in favour of scheme "A," but the governing bodies of The London, St. Thomas's and The Royal Free had already committed themselves to the Sussex scheme before the meeting. It is obvious that such schemes as the "Oxford" or "Sussex" scheme would not be satisfactory for the ophthalmic hospitals of London. First, because the ophthalmic hospitals of London supply a far wider area than London itself; secondly, because there could be no way of defining the areas from which contributions could be made to each individual hospital, and thirdly, that at any rate the "Sussex" 
scheme embodies in it a certain amount of contract practice, in that the staff of the hospitals receive 25 per cent. of the sum collected. We think it extremely undesirable that the character of the institutions should be thus changed and that the staff who give their services voluntarily should become paid servants of the hospital, and we are wondering if the staffs of the three great hospitals above-mentioned realize the conditions which the adoption of this scheme entails.

\section{Medical Advice to the Public}

Medical Men who give gratuitous advice to the public should be very careful as to the accuracy of their statements. In a recent publication on "Venereal Diseases and their Effects," by the National Council for Combating Venereal Diseases, there is a paragraph on the "Effect of Gonorrhoea on Offspring." It is there stated that " a child born to a woman suffering from gonorrhoea runs a grave risk; during birth, of receiving the infection in the. eyes. The result is the development a few days after birth of an acute inflammation of the eyes (ophthalmia neonatorum), which is a very frequent source of complete blindness." The writer goes on to state, that "This disease can be prevented if drops of 1 per cent. protargol are instilled into the child's eyes immediately after birth." As it stands this statement is grossly inaccurate, and even if there has been a printer's error in the strength of the solution, the statement is open to grave doubt. That prophylactic treatment with drugs has proved beneficial has been shown by Crede's treatment-in which, as a matter of fact, 1 per cent. silver nitrate was used. The routine use of such methods, however, by midwives and others, is liable to cause a false sense of security (which would certainly be increased by the above statement), and is furthermore liable to misuse and serious mistakes. Thus, we have heard of a case in which strong nitric acid was dropped into a baby's eye in mistake for a silver preparation.

In any case, seeing that presumably these pamphlets of the National Council are not circulated among medical men only, we regard it as highly undesirable that any instructions as to treatment with drugs should be given. It would have been wiser to have laid stress upon the supreme importance of immediately referring cases of this nature to treatment by competent medical men. 